\title{
Severe pneumonia due to an atypical organism in an immune-competent patient
}

\author{
Kavita Pal ${ }^{1}$, Ankur Girdhar ${ }^{2}$, Abubakr Bajwa ${ }^{2}$ \\ 1. Department of Internal Medicine, UF College of Medicine, Jacksonville, USA. 2. Division of Pulmonary and Critical care, \\ UF College of Medicine, Jacksonville, USA.
}

Correspondence: Kavita Pal. Address: Department of Internal Medicine, UF College of Medicine at Jacksonville 655, West 8th street, Jacksonville, FL 32209, USA. Email: kavita.pal@jax.ufl.edu

Received: March 2, 2015

DOI : $10.5430 /$ crim.v2n2p76
Accepted: March 29, $2015 \quad$ Online Published: April 27, 2015

URL: http://dx.doi.org/10.5430/crim.v2n2p76

\section{Abstract}

Myroides species, formerly classified as Flavobacterium odoratum, is a waterborne pathogen. It is well known to cause infection in immune-compromised patients. Infection by this organism is acquired occupationally or nosocomial via inhalation, ingestion or dermal absorption. Because these organisms are uncommon they may be difficult to identify and treat promptly. We present a case of a young immune-competent fish farm worker who presented with typical pneumonia like symptoms but eventually having an overwhelming inflammatory cascade secondary to an atypical infection by Myroides species.

\section{Keywords}

Myroides, Flavobacterium odoratum, Myroid, Immune-competent, Pneumonia, Atypical infection

\section{Introduction}

The genus Myroids, which was formerly classified as Flavobacterium odoratum was initially described in early 20th century. Myroid strains have been isolated clinically from human by products such as urine, feces, wound discharge, sputum, and blood. It mostly causes a wide spectrum of cutaneous illnesses. Only recently cases of disseminated infections have been reported. Most of the systemic manifestation due to Myroid is seen in the immune-compromised patients as Myroids usually behaves as a low grade opportunistic infection. We report a case of severe pneumonia and sepsis caused by $\mathrm{M}$. odoratimimus infection.

\section{Case presentation}

A 27 year old male, with no significant medical or surgical history, presented to our hospital with progressive shortness of breath for two weeks associated with cough and yellow colored expectoration. He also reported subjective fevers and a recent unintentional 10-pound weight loss. He denied night sweats, chest pain, hemoptysis or diarrhea. Patient had been smoking half packet of cigarettes per day for the last 7 years. His primary occupation was as a worker on a fish farm which involved direct handling of fish. Careful history taking revealed no recent contact with the ill, high risk sexual behavior or travel inside or outside the country. 
Clinical examination revealed a thin, frail, lethargic young male, with dry mucous membranes. Lung examination was significant for coarse rales present diffusely and diminished bibasilar breath sounds. He was tachycardic, and had bilateral lower extremity pitting edema. Skin examination was remarkable for fissures on bilateral palms, which appeared chronic in nature and reported as per the patient to be as a result of contact with water and fish.

His laboratory tests showed a white blood cell count of $4500 / \mathrm{mm}^{3}$, hemoglobin of $10.5 \mathrm{gram} / \mathrm{dl}$, platelet count of $60,000 / \mathrm{mm}^{3}$, international normalized ratio was elevated at 3.5, plasma sodium of $130 \mathrm{mmol} / \mathrm{L}$, bicarbonate level of 18 $\mathrm{mmol} / \mathrm{L}$ and a lactic acid level of $5.9 \mathrm{mmol} / \mathrm{L}$. His arterial blood gas showed a $\mathrm{pH}$ of 7.04, $\mathrm{pCO}_{2}$ of $63 \mathrm{mmHg}$ and a pO $\mathrm{O}_{2}$ of $71 \mathrm{mmHg}$ on a $100 \% \mathrm{FiO}_{2}$. The rest of his metabolic panel was within normal range including his renal function. He was found to be HIV negative by ELISA. His initial chest X-Ray (see Figure 1) revealed a large right sided pleural effusion with patchy parenchymal opacities bilaterally.

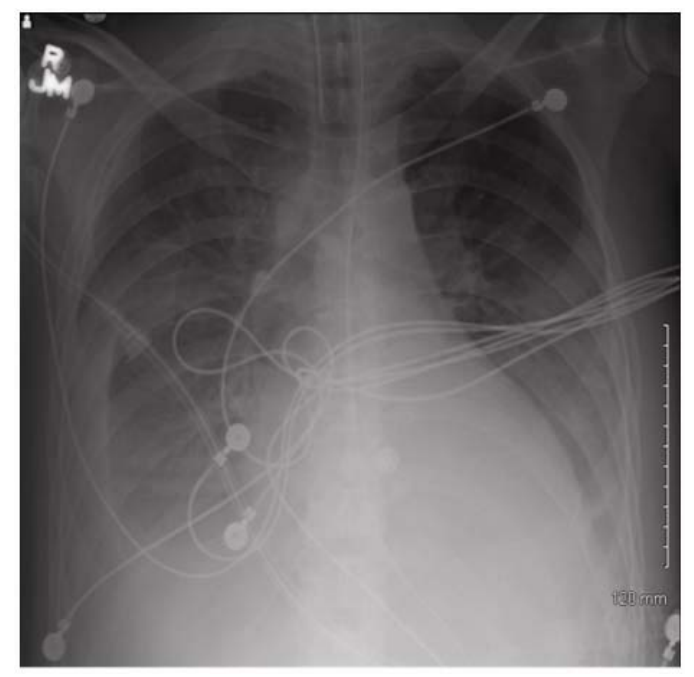

Figure 1. Patient's chest X-ray at the time he was admitted to the ICU, showing right sided effusion with bilateral patchy opacities and air bronchograms.

At this time patient was admitted to the hospital with a diagnosis of severe community acquired pneumonia in an immunecompetent patient complicated with disseminated intravascular coagulopathy, hyponatremia and possible para-pneumonic effusion.

Shortly after his admission, the patient became increasingly altered with worsening work of breathing and tachycardia. He required intubation for acute respiratory failure and also became hypotensive eventually requiring vasopressor support. He was transferred to the intensive care unit (ICU) with the additional diagnosis of septic shock secondary to community acquired pneumonia and was started on empiric antibiotic therapy. A computed tomography (CT) chest scan (see Figure 2) was done to better visualize the pleural effusion, which showed multifocal patchy airspace and ground glass opacities in the bilateral lungs, concerning for multifocal pneumonia and a right sided large non-loculated pleural effusion. A transthoracic echocardiogram showed severe left ventricular dysfunction and a low left ventricular ejection fraction of $15 \%-20 \%$ with no visible valvular vegetation.

The patient was initially started on ceftriaxone and azithromycin for community acquired pneumonia, but after 48 hours of treatment there was no obvious clinical improvement noted. His antibiotics were changed to vancomycin, piperacillintazobactam and levofloxacin to cover for multi-drug resistant organisms. Despite the use of these broad spectrum antibiotics patient continued to require vasopressors and full ventilator support with minimal signs of clinical improvement. An extensive diagnostic panel to identify the source of infection was performed including the work up for atypical organisms (see Table 1). Simultaneously a pleural tap was done which was consistent with an exudative effusion 
and on gram staining showed gram negative rods. Both broncho-alveolar lavage and repeat blood cultures performed at this time revealed Myroid spp. of the odoratimimus species. This organism had in-vitro sensitivity to Meropenem. The antibiotics were changed accordingly, and within 48 hours the patient rapidly improved. His vasopressor requirement drastically reduced with improvement in his chest X-rays. The patient required a percutaneous tracheostomy but was eventually weaned off of ventilator support to be discharged back home with a 4 week course of the antibiotic.

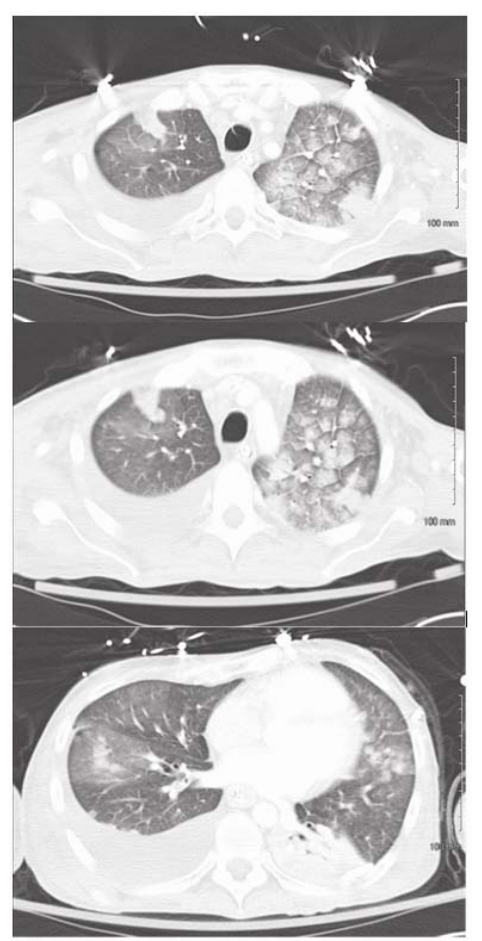

Figure 2. Representative images of the CT chest of the patient showing right sided moderate sized effusion with bilateral patchy ground glass opacities and surrounding thickened interlobular septa.

Table 1. Extensive diagnostic panel to identify the source of infection

\begin{tabular}{|c|c|c|c|}
\hline ID & & Auto-immune panel & \\
\hline Coxsackie B 1-6 Antibody & Negative & ANA & Negative \\
\hline Coxsackie A Immunoglobulin G & Negative & C-ANCA & $<1: 20$ \\
\hline Coxsackie A Immunoglobulin $\mathrm{M}$ & Negative & P-ANCA & $<1: 20$ \\
\hline Legionella Antigen & Negative & Atypical P-ANCA & $<1: 20$ \\
\hline Streptococcus pneumoniae Antigen & Negative & Rheumatoid Factor & Negative \\
\hline Ehrlichia chaffeensis PCR & Negative & & \\
\hline Acid Fast Bacilli stain & Negative & Hepatitis Panel & \\
\hline Histoplasma Antigen & 2.98 & Hepatitis A Immunoglobulin M & Negative \\
\hline Leptospira Antibody & Negative & Hepatitis B Immunoglobulin M & Negative \\
\hline Parvovirus B19 Immunoglobulin M & 0.2 & Hepatitis Surface Antigen & Negative \\
\hline West Nile Virus Immunoglobulin M/ Immunoglobulin G & Negative & Hepatitis C Antibody & Negative \\
\hline Herpes Simplex Virus 1/2 DNA PCR & Negative & & \\
\hline Influenza A/B viral PCR & Negative & HIV Testing & \\
\hline Adenovirus/RSV/Parainfluenza 1,2,3 PCR & Negative & HIV-1 RNA Viral Load & Not Detected \\
\hline H1N1 (2009) PCR & Negative & HIV $1 / 2$ Antibody & Negative \\
\hline Lyme disease PCR & Negative & & \\
\hline
\end{tabular}

Note. PCR: Polymerase Chain Reaction, ANA: Anti-nuclear antibody, C-ANCA: Cytoplasmic Anti-neutrophil cytoplasmic antibody, P-ANCA: Perinuclear Anti-neutrophil cytoplasmic antibody, HIV: 


\section{Discussion}

The genus Myroids, formerly classified as Flavobacterium odoratum, was first isolated in $1923^{[1]}$. Myroid strains have been primarily isolated clinically, however, are also commonly found in soil and water. A number of studies have shown that this organism has a predilection for aquatic environments ${ }^{[2,3]}$.

The organism has been isolated from urine, blood, wounds, and respiratory secretions ${ }^{[4,5]}$. Clinical infection with Myroides spp. is rare and it usually behaves as a low grade opportunistic infection. There are two species identified within the genus: Myroides odoratus and Myroides odoratimimus.

Cases of endocarditis ${ }^{[6]}$, ventriculitis ${ }^{[7]}$, soft tissue infections ${ }^{[8]}$, urinary tract infection ${ }^{[4]}$ and central venous catheterassociated bloodstream infection ${ }^{[9]}$ due to contaminated water in the hospital air circulation and ventilator circuits, have been reported. Molecular typing techniques have allowed for discrimination of the causative species and it has been found that M. odoratus as the etiological agent in cases of septic cellulitis and M. odoratimimus as responsible for cases of urinary tract infections and two cases of bacteremia ${ }^{[8,10]}$. Most patients described in these cases have been severely immune-compromised patient. To our knowledge there is only one other case reported with M. Odoratimimus in a young immune-competent male ${ }^{[11]}$ with a similar presentation as our patient, but had a different occupation and with the inciting event being massive trauma and skin contamination.

The species from this genus are able to colonize ubiquitous environments by producing surface active compounds (cholic acid, deoxycholic acid, and their conjugates with glycine) ${ }^{[12]}$ giving them the capacity to invade deeper mucosa and eventually disseminate by means of the blood stream. M. odoratus isolates were also shown to display strong adherence profiles, with a preference at lower temperatures (about $21^{\circ} \mathrm{C}$ ) and nutrient-rich environments with low hydrodynamic forces ${ }^{[13]}$.

Species of this genus also can form bio-films which help protect more pathogenic organisms from disinfectants and antibiotics. In fact, M. odoratus strains can contribute significantly to the attachment of Listeria monocytogenes to stainless steel food surfaces and adds to the resistance of this organism to eradication by chlorine ${ }^{[14]}$. These characteristics could explain their wide distribution in the environment. Often times, the water in the hospital environment is suspected of carrying the organisms ${ }^{[15]}$.

The broad resistance to antibiotics is characteristic of Myroides spp. Most strains are resistant to aminoglycosides and beta-lactams including Imipenem by the production of a chromosome-encoded metallo-beta-lactamase (TUS-1 and MUS-1) ${ }^{[16]}$. However, these strains have also demonstrated resistance independent of TUS-1 and MUS-1 ${ }^{[17]}$ which warrants further investigation. The particular strain in our patient was resistant to the initial antibiotic regimen leading to his clinical deterioration. Accurate and reliable susceptible testing allowed us to promptly change the antibiotics to Meropenem to appropriately treat the patient. This highlights the importance of prompt isolation of organisms and obtaining sensitivity analysis for antibiotic coverage.

Myroids spp was once considered as an opportunistic pathogen and a rare cause of serious disease even in heavily immune-compromised patients. Recent case reports, including ours, suggest that organisms from this genus can cause severe life threatening diseases even in immune-competent hosts ${ }^{[11]}$.

Our patient was a young and healthy male with no clinical risk factors, presenting with an overwhelming inflammatory cascade secondary to a Myroides infection. Nosocomial transmission to our patient cannot be excluded, however, given his occupational history it is likely that he was exposed to the pathogen via inhalation, ingestion or dermal absorption route. Moreover, during his hospitalization the organism was not isolated from any other patient admitted into the ICU of our hospital. 
Waterborne pathogens, although uncommon, are known to cause cutaneous infections. More than 100 species of pathogenic bacteria, viruses, and protozoa can be identified in contaminated water ${ }^{[18]}$. Often times, these infections are acquired via occupational or recreational means. Exposures include inhalation, ingestion or dermal absorption of these organisms. Because these organisms are uncommon they may be difficult to identify and treat promptly.

A detailed occupational history of patients is crucial to the differential diagnosis and treatment of infections. Our patient had an occupational hazard to developing this rare opportunistic infection. Prompt diagnosis and treatment in young and healthy patients allows for a rapid recovery and shorter ICU length of stay.

\section{Conclusion}

Our case highlights that immune-competent patients with typical pneumonia like presentation can have an atypical cause such as Myroids spp. M. odoratimimus should be considered a serious and life threatening nosocomial pathogen and it is important for physicians to keep a low threshold for diagnosis of this organism especially in patients with occupational exposures, skin infection or major trauma.

\section{References}

[1] Vancanneyt M, Segers P, Torck U, et al. Reclassification of Flavobacterium odoratum (Stutzer 1929) strains to a new genus, Myroides, as Myroides odoratus comb. nov. and Myroides odoratimimus sp nov Int J Syst Bacteriol. 1996; 46: 926-32. http://dx.doi.org/10.1099/00207713-46-4-926

[2] Yoon J, Maneerat S, Kawai F, et al. Myroides pelagicussp. nov., isolated from seawater in Thailand. nt J Syst Evol Microbiol. 2006; 56: 1917-20. PMid:16902031 http://dx.doi.org/10.1099/ijs.0.64336-0

[3] Cho SH, Chae SH, Im WT, et al. Myroides marinussp. nov., a member of the family Flavobacteriaceae, solated from seawater. Int J Syst Evol Microbiol. 2010 May; 21.

[4] Yağci A, Cerikcioğlu M. E, Kaufmann H, et al. Pitt Molecular typing of Myroides odoratimimus (Flavobacterium odoratum) urinary tract infections in a Turkish hospital Eur J Clin Microbiol Infect Dis. 2000; 19: 731-2. PMid:11057514 http://dx.doi.org/10.1007/s100960070001

[5] Holmes B, Snell J. J. Lapage Flavobacterium odoratum: a species resistant to a wide range of antimicrobial agents J Clin Pathol. 1979; 32: 73-7. PMid:429582 http://dx.doi.org/10.1136/jcp.32.1.73

[6] Ferrer C, Jakob E, Pastorino G. Juncos Right-sided bacterial endocarditis due to Flavobacteriun odoratum in a patient on chronic haemodialysis Am J Nephrol. 1995; 15: 82-4. PMid:7872370 http://dx.doi.org/10.1159/000168806

[7] MacFarlane D. E, Baum-Thureen P. I. Crandon Flavobacteriun odoratum ventriculitis treated with intraventricular cefotaxime J Infect. 1985; 11: 233-8. http://dx.doi.org/10.1016/S0163-4453(85)93228-1

[8] Bachmeyer C, Entressengle H, Khosrotehrani K, et al. Cellulitis due to Myroides odoratimimus in a patient with alcoholic cirrhosis. Clin Exp Dermatol. 2008; 33: 97-8. PMid:18039344

[9] Douce RW, Zurita J, Sanchez O, et al. Investigation of an outbreak of central venous catheter-associated bloodstream infection due to contaminated water. Infect Control Hosp Epidemiol. 2008; 29: 364-6. PMid:18462150 http://dx.doi.org/10.1086/533543

[10] Bachman K. H, Sewell D. L, Straubaugh L. J. Recurrent cellulitis and bacteremia caused by Flavobacterium odoratum. Clin Infect Dis. 1996; 22: 1113-14. http://dx.doi.org/10.1093/clinids/22.6.1112

[11] Benedetti P, Rassu M, Pavan G, et al. Septic shock, pneumonia, and soft tissue infection due to Myroides odoratimimus: report of a case and review of Myroides infections. Infection. 2011; 39: 161-5. PMid:21246247 http://dx.doi.org/10.1007/s15010-010-0077-1

[12] Maneerat S, Nitoda T, Kanzaki H, et al. Bile acids are new products of a marine bacterium, Myroides sp. strain SM1. Appl Microbiol Biotechnol. 2005; 67: 679-83. PMid:15549287 http://dx.doi.org/10.1007/s00253-004-1777-1

[13] Jacobs A, Chenia HY. Biofilm-forming capacity, surface hydrophobicity and aggregation characteristics of Myroides odoratus isolated from South African Oreochromis mossambicus fish. J Appl Microbiol. 2009; 107: 1957-66. PMid:19519668 http://dx.doi.org/10.1111/j.1365-2672.2009.04380.x

[14] Bremer PJ, Monk I, Butler R. Inactivation of Listeria monocytogenes/Flavobacterium spp. biofilms using chlorine: impact of substrate, $\mathrm{pH}$, time and concentration. Lett Appl Microbiol. 2002; 35: 321-5. PMid:12358696 http://dx.doi.org/10.1046/j.1472-765X.2002.01198.x 
[15] Hugo CJ, Bruun B, Jooste PJ. The genera Empedobacter and Myroides. In: Dworkin M, Falkow S, Rosenberg E, Schleifer K-H, Stackebrandt E, editors. The prokaryotes, vol. 7. 3rd ed. New York: Springer; 2006. 630-7 p. http://dx.doi.org/10.1007/0-387-30747-8_24

[16] Mammeri H, Bellais S, Nordmann P. Chromosome-encoded $\beta$-lactamases TUS-1 and MUS-1 from Myroides odoratus and Myroides odoratimimus (formerly Flavobacterium odoratus), new members of the lineage of molecular subclass B1 metalloenzymes. Antimicrob Agents Chemother. 2002; 46: 3561-7. PMid:12384365 http://dx.doi.org/10.1128/AAC.46.11.3561-3567.2002

[17] Dharne M. S, Gupta A. K, Rangrez A.Y, et al. Antibacterial activities of multi drug resistant Myroides odoratimimus bacteria isolated from adult flesh flies (Diptera: sarcophagidae) are independent of metallo beta-lactamase gene. Braz J Microbiol. 2008 Apr-Jun; 39(2): 397-404. PMid:24031236 http://dx.doi.org/10.1590/S1517-83822008000200035

[18] Rose J B, Epstein P R, Lipp E K, et al. Climate variability and change in the United States: potential impacts on water- and foodborne diseases caused by microbiologic agents. Environ Health Perspect. May 2001; 109(Suppl 2): 211-21. PMid:11359688 http://dx.doi.org/10.2307/3435011 